\title{
Fixed-speed and Variable-speed Pumped Storage Dispatch Model in Power Systems with High Renewable Penetration
}

\author{
Bo Yuan ${ }^{1, *}$, Jin Zong ${ }^{2}$, and Zhicheng $\mathrm{Xu}^{1}$ \\ ${ }^{1}$ State Grid Energy Research Institute, Beijing, 102209, China \\ ${ }^{2}$ State Grid Jibei Electric Power Company, Beijing, 10020, China
}

\begin{abstract}
According to different operating characteristics of pumped storage fixed speed unit and variable speed unit, a joint dispatching model of pumped storage unit and other types of units based on mixed integer linear optimization is constructed. The model takes into account the operating conditions, reservoir capacity, cycle type and other pumped storage unit constraints, but also consider the frequent start and stop and the stability of the operation of the unit caused by the loss. Using the Cplex solver to solve the model, the empirical example of the provincial power grid shows that the model can effectively arrange the pumping storage speed and the dispatching operation of the variable speed unit under the precondition of economic life of the unit, and give full play to the function of peak shaving and accommodating new energy. Because of its more flexible regulation characteristics of power generation and pumping conditions, the variable speed unit can better improve the operating conditions of other units in the system and promote the new energy dissipation.
\end{abstract}

\section{Introduction}

Pumped storage power station is the most mature energy storage system in power system and a flexible power supply with excellent performance. It is an effective means to solve the problem of power system peak regulation, reduce new energy discard and ensure the safe and stable operation of the system.

The traditional fixed-speed pumped storage unit can only operate at rated power at fixed speed, and it may appear "overshoot peak" phenomenon in the period of low load, and even need to start or increase output of thermal power unit to balance the difference between pumping output and breathing energy., and the variable speed unit can be more flexible to cope with the load and intermittent power fluctuations because of the stable operating range under the pumping conditions, and the related scheduling model and scheduling strategy are also different. In [1], a low carbon scheduling model of pumped-storage variable speed unit is established by using a mixed integer quadratic programming model and a minimum cost flow algorithm. The scheduling strategy of pumped-storage variable speed unit is discussed beneficially; however, the quadratic programming model needs further improvement due to its long solving complexity and time.

In this paper, based on mixed integer linear programming model, the scheduling model of fixed speed and variable speed unit of pumped storage power station is established, and the loss caused by frequent start and stop of pumped storage unit and the operation of stability zone is fully considered. By using the Cplex and analyzing the example of the provincial power grid, the difference of the scheduling result between the variable speed and the fixed speed unit is compared, and the validity of the proposed model is validated. The results show that the model can arrange the speed of pumped storage and the operation mode of variable speed unit reasonably, and give full play to its peak regulation, promote the new energy consumption and reduce the operating cost of the system under the premise of considering the economic life of the unit.

\section{Model of Pumped Storage Unit in Optimal Dispatching}

\subsection{Output Range Constraint}

Under the condition of generating mode, the output range of fixed speed and variable speed pumped storage unit is

$$
U_{\text {pump }} P_{g, \text { min }} \leq P_{g} \leq U_{\text {pump }} P_{g, \text { max }}
$$

Where $U_{\text {pump }}$ is the working condition of pumped storage, 1 represents generating mode and 0 represents pumping mode. $P_{g, \text { min }}$ and $P_{g, \text { max }}$ are the minimum and maximum output range of generating mode.

Under the condition of pumping mode, for variablespeed pumped storage, we give

$$
\left(1-U_{\text {pump }}\right) P_{p, \min } \leq P_{p} \leq\left(1-U_{\text {pump }}\right) P_{p, \text { max }}
$$

Where $P_{p, \min }$ and $P_{p, \max }$ are the minimum and maximum output range of pumping mode.

\footnotetext{
* Corresponding author: yuanbo@sgeri.sgcc.com.cn
} 
For fixed-speed pumped storage, we give

$$
P_{p}=\left(1-U_{\text {pump }}\right) P_{p, \max }
$$

Which means the output under pumping mode can only be 0 or rated power.

\subsection{Reservoir Capacity Constraint}

When a pumped storage unit is in operation, the water level needs to be maintained between the maximum water level and the dead water level.

$$
\begin{gathered}
W_{u, \text { min }} \leq W_{u} \leq W_{u, \text { max }} \\
W_{d, \text { min }} \leq W_{d} \leq W_{d, \text { max }}
\end{gathered}
$$

Where $W_{u}$ and $W_{d}$ are the water level of the upper and the lower reservoir; $W_{u, \min }$ and $W_{u, \max }$ are the minimum and maximum water level of the upper reservoir. $W_{d, \min }$ and $W_{d, \max }$ are the minimum and maximum water level of the lower reservoir.

\subsection{Water Level Dynamic Equilibrium}

$$
\begin{aligned}
& W_{u}^{t}=W_{u}^{t-1}-\lambda_{g} P_{g}^{t}+\lambda_{p} P_{p}^{t} \\
& W_{d}^{t}=W_{d}^{t-1}+\lambda_{g} P_{g}^{t}-\lambda_{p} P_{p}^{t}
\end{aligned}
$$

Where $\lambda g$ is the generating conversion coefficient and $\lambda_{p}$ is the pumping conversion coefficient.

\subsection{Daily/weekly Reservoir Cycle}

For the day/week cycle of reservoir scheduling type units, assuming that one day/week is divided into $s$ slices, the initial and final period of the upper reservoir water level should be consistent.

$$
W_{u}^{s}=W_{u}^{0}
$$

Where $W_{u}^{0}$ is the water level of initial time period and $W_{u}^{s}$ is the water level of final time period.

\subsection{Overcall Losses of Generating Units}

The output range of pumped storage unit can be divided into stable operation zone $\left(P_{p \text {,stable }}\right.$ and $\left.P_{g \text {,stable }}\right)$ and minimum technical output zone $\left(P_{p, \min }\right.$ and $\left.P_{g, \min }\right)$, which can reflect the unit loss caused by the carrying load outside the stable operation zone. An auxiliary binary decision variable is introduced to represent the operating zone. $k=1$ represent the pumping mode stable operation zone, $k=2$ represent the pumping mode minimum technical operation zone, $k=3$ represent shut down, $k=$ 4 represent the generating mode minimum technical operation zone, $k=5$ represent the generating mode stable operation zone.

A continuous decision variable is also introduced to represent the output in each operation zone. The following constraints should be met.

$$
\sum_{k} w_{i, k}^{t}=1
$$

$$
\begin{gathered}
\sum_{k} P_{\text {pump }, k}^{t}=P_{p}+P_{g} \\
w_{i, 3}^{t}=1-U_{\text {pump }}^{t} \\
P_{\text {pump }, k \text { min }} w_{i, k}^{t} \leq P_{\text {pump }, k}^{t} \leq P_{\text {pump }, k \max } w_{i, k}^{t}
\end{gathered}
$$

(9)guarantees that each output state is mutually exclusive; (10) (11) makes the auxiliary decision variable and the output range of the original decision variable coupled; (12) is the upper and lower bound of output in each operation zone.

After the auxiliary decision variable is introduced, two methods can be used to deal with the loss modeling of operating outside the stable operation zone. One is to specify in the constraint condition that the running time outside the stable operation zone of the unit per day cannot be greater than the given value $t_{\min }$, which is

$$
\sum_{t}\left(w_{i, 2}^{t}+w_{i, 4}^{t}\right) \leq t_{\min }
$$

The other method is the operation loss cost function for a given unstable operating region, which is included in the objective function of the scheduling model as follow

$$
C_{\text {pump }}=h \cdot \sum_{t}\left(P_{\text {pump }, 4}^{t}-P_{\text {pump }, 2}^{t}\right)
$$

\begin{tabular}{|c|c|c|}
\hline & Type & Illustraion \\
\hline$P_{\text {withpi }}^{t}$ & $\begin{array}{l}\text { continuou } \\
\text { s }\end{array}$ & the output of unit $i$ in period $t$ \\
\hline$U_{\text {thermal }, i}^{t}$ & binary & on/off state of unit $i$ in period $t$ \\
\hline$P_{g, i}^{t}$ & $\begin{array}{c}\text { continuou } \\
\mathrm{S}\end{array}$ & $\begin{array}{c}\text { generating output of pumped storage } \\
\text { unit }\end{array}$ \\
\hline$P_{p, i}^{t}$ & $\begin{array}{l}\text { continuou } \\
\mathrm{S}\end{array}$ & $\begin{array}{c}\text { pumping output of pumped storage } \\
\text { unit }\end{array}$ \\
\hline$U_{p u m p, i}^{t}$ & binary & $\begin{array}{c}\text { operating mode pumped storage unit, } \\
1 \text { represent generating and } 0 \\
\text { represent pumping }\end{array}$ \\
\hline
\end{tabular}

Where $h$ is the cost coefficient of operating outside the stable zone.

\section{Optimal Dispatching Model of the System}

\subsection{Decision Variables}

The decision variables include the output of thermal power, hydropower, wind power, solar energy, pumped storage unit, and the on/off status of the thermal units, and the operating conditions of pumped storage units.

Table 1. Decision Variables of the Dispatching Model

\subsection{Objective Function}

The objective function is the minimum operating cost of the system:

$$
C=\sum_{t} \sum_{i} C_{i}^{S U}\left(1-U_{\text {themal }, i}^{t-1}\right) U_{\text {thermal }, i}^{t}+\sum_{t} \sum_{i} F\left(P_{\text {thermal }, i}^{t}\right)+C_{p \text { pump }}
$$


Where $C_{i}^{S U}$ is the start-up cost of thermal unit $i ; F()$ is the fuel cost function of thermal unit; $C_{p u m p}$ is the overcall operation loss cost function of pumped storage.

\subsection{Constraints}

(1) Power balance constraint

$$
\forall t, \sum_{s \in \text { unittype }} \sum_{i} P_{s, i}^{t}+\sum_{i} P_{g, i}^{t}-\sum_{i} P_{p, i}^{t}=L_{t}
$$

Where $L_{t}$ is the load demand in period $t$.

(2) Maximum and minimum output range

$$
\forall t, \forall i, \forall s \in \text { unittype, } \quad P_{s, i, \min } \leq P_{s, i}^{t} \leq P_{s, i, \text { max }}
$$

Where $P_{s, i, \text { min }}$ and $P_{s, i, \max }$ are the maximum and minimum output of unit $i$. For wind and solar power, the maximum output at each moment is the predicted available output at that moment.

(3) Minimum on/off time constraint

$$
\begin{array}{r}
\sum_{l=t}^{t+T_{\text {ion }}-1} U_{s, i}^{l} \geq T_{i, o n}\left(U_{s, i}^{l}-U_{s, i}^{l-1}\right) \\
\sum_{l=t}^{t+T_{\text {iog }}{ }^{-1}}\left(1-U_{s, i}^{l}\right) \geq T_{i, o n}\left(U_{s, i}^{l-1}-U_{s, i}^{l}\right)
\end{array}
$$

Where $T_{i, o n}$ and $T_{i, o f f}$ are the minimum on and off time of unit $i$.

(4) Ramp rate constraint

$$
\begin{aligned}
P_{s, i}^{t} & \geq P_{s, i}^{t-1}-R D_{s, i}-\left(1-U_{s, i}^{t}\right) P_{s, i, \max } \\
P_{s, i}^{t} & \leq P_{s, i}^{t-1}+R U_{s, i}+\left(1-U_{s, i}^{t-1}\right) P_{s, i, \max }
\end{aligned}
$$

Where $R D_{s, i}$ and $R U_{s, i}$ are the up and down ramp rate of unit $i$.

(5) Spinning reserve constraint

$$
\begin{gathered}
\sum_{s} \sum_{i} R_{s, i}^{t} \geq L_{t}\left(1+R_{L}\right) \\
\sum_{s} \sum_{i} R_{s, i}^{t} \leq L_{t}\left(1+R_{V G}\right)
\end{gathered}
$$

Where $R_{L}$ and $R_{V G}$ are the reserve requirement of load and variable generations; $R_{s, i}^{t}$ are the available spinning reserve provided by unit $i$.

(6) Pumped storage constraint

The pumped storage constraints are (1)-(14).

\section{Case Study}

\subsection{Test System}

The objective function of the proposed model involves that the starting and stopping costs of thermal power units and pumped storage units are quadratic functions, which can be converted into linear functions according to the method in [15]; In this case, the whole model is a mixed integer linear optimization model, which can be programmed by using OPL language developed by IBM and solved by CPLEX.
Based on the 2030-year planning system of a real power grid in the eastern province, this paper studies a typical day 24-hour power system dispatch situation. The typical daily system load curve is shown in Fig. 2.

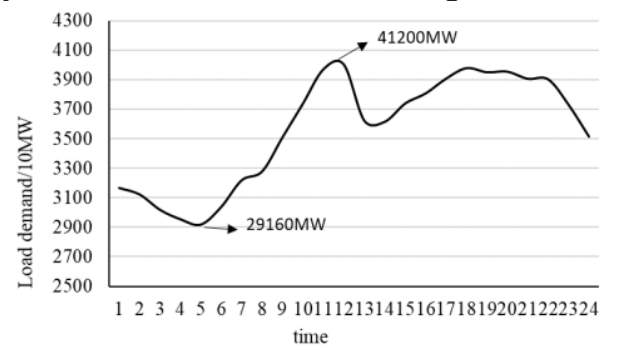

Fig. 1. Load Curve

\subsection{Results}

If there is no pumped-storage power station in the system, the unit output arrangement is shown in Figure 3, and the wind/solar curtailment is shown in Table 3. Wind/solar curtailment is 66 million $\mathrm{Kwh}$, the curtailment rate is $24.2 \%$.

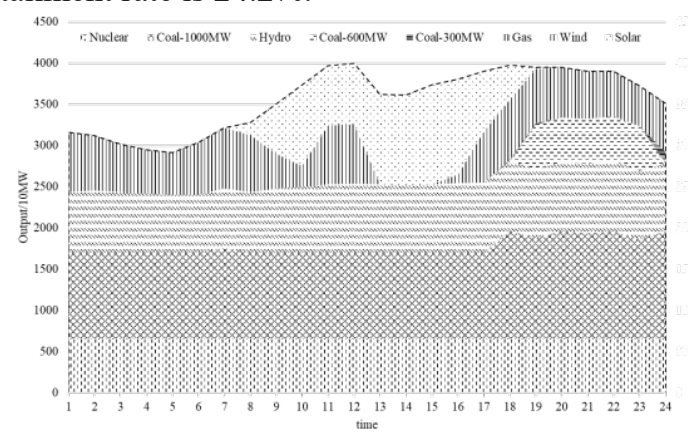

Fig. 2. Dispatch result without pumped storage

Eight fixed speed pumped storage units of 300,000 $\mathrm{kW}$ were added to the system, unit loss is not take into consideration, and the dispatch result is shown in Figure 3. Results shows that the pumped storage unit can pump water during the period of low net load and generate electricity during the peak period, so that the tasks of peak regulation and new energy consumption can be well realized. After adding a fixed-speed pumped storage unit, the total amount of the system curtailment is about 54.43 million $\mathrm{kw} \mathrm{h}$, and the curtailment rate is $19.9 \%$, which is $4.3 \%$ lower than the system without pumped storage.

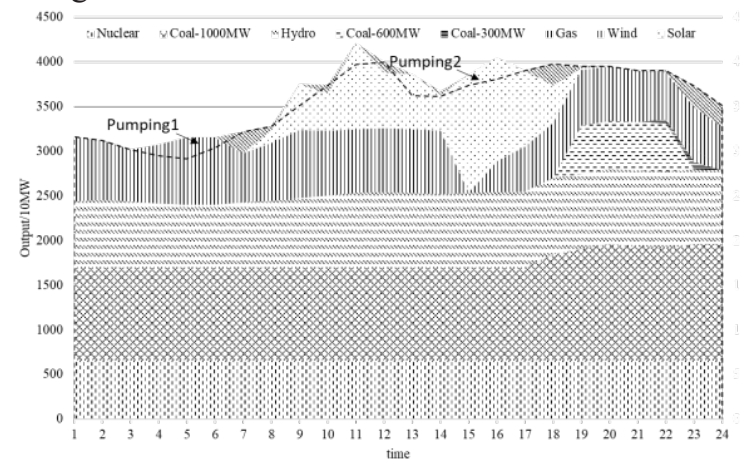

Fig. 3. Dispatch result with fixed-speed pumped storage

The fixed speed unit is replaced by variable speed unit of the same capacity. The model also arranges the output of the variable speed unit well, effectively 
realizes the peak shaving and new energy consumption function; at the same time, due to the stepless adjustment of pumping conditions, the system operation is more flexible, the amount of curtailment is reduced to 46 million $\mathrm{kW} \mathrm{h}$, and the curtailment rate is $16.9 \%$.

When taking into account the loss of pumped storage, the total cost of the system will rise, but the operating cost of the system is still lower than that of no pumped storage, see table 2;

Table 2. Curtailment and utilization hour of pumped storage in different scenarios

\begin{tabular}{cccc}
\hline & & $\begin{array}{c}\text { Curtailme } \\
\text { nt/\% }\end{array}$ & $\begin{array}{c}\text { Daily } \\
\text { utilization } \\
\text { hour/hour }\end{array}$ \\
\hline no pumped storage & 24.2 & - \\
\hline $\begin{array}{c}\text { no } \\
\text { loss }\end{array}$ & $\begin{array}{c}\text { fixed-speed } \\
\text { variable- } \\
\text { speed }\end{array}$ & 19.9 & 5.2 \\
& 16.9 & 6.5 \\
loss & $\begin{array}{c}\text { fixed-speed } \\
\text { variable- }\end{array}$ & 20.6 & 4.3 \\
& speed & 17.3 & 6.0 \\
\hline
\end{tabular}

From table 2, it can be found that the operating cost of the system and the curtailment rate of are slightly increased, but the operating range is controlled in a better interval. This shows that the unit can operate in a more secure and stable range after considering the excessive call of pumped storage.

\section{Conclusion}

In this paper, the loss model of pumping storage with operating outside stable zone is constructed, the joint dispatch model of pumped storage and other types of units based on mixed integer linear programming is built, and the model is solved using Cplex, considering the various operating characteristics of fixed-speed and variable-speed unit. Through to the provincial power grid demonstration analysis obtains the following conclusion:

(1) The proposed model can effectively arrange the output of fixed-speed and variable-speed pumped storage unit under the premise of considering the economic life of the unit, give full play to the function of peak shaving and new energy consumption;

(2) In terms of promoting new energy consumption, the variable speed unit has stepless regulation characteristics due to its large working range of power generation and pumping condition, which makes the system run more flexibly and can effectively solve the phenomenon of "over peak shaving" of the fixed speed pumping unit, better improve the operating conditions of other units in the system and reduce curtailment.

(3) After taking into account the overcall loss, the pumped storage unit can operate in a safer and more stable zone. Although the system curtailment has increased slightly in the short term, in the long run, the failure frequency and maintenance time of the pumped storage unit have been reduced, the life of the unit has been extended, and the unit can be better guaranteed to give full play of the new energy consumption and peakshaving function.

\section{Acknowledgements}

This work is supported by Science and Technology Project of State Grid Corporation of China (B3670116A005) : Study on the evaluation model and application of peak shaving technology, economy and policy for power system wiht large-scale renewable energy integration.

\section{References}

1. Bueno C., Carta J. A.. Technical-economic Analysis of Wind powered Pumped Hydro storage Systems. Part 2: Model Application to the Island of EI Hierro [J]. Solar Energy, 2005, 78(3): 396-405.

2. Kenneth B, Yury D, Erik D, et al . Coupling pumped hydro energy storage with unit commitment[J] . IEEE Tansactions on Sustainable Energy, 2016, 7(2) : 786-796

3. Garcia-Gonzalez J., de la Muela R. M. R., Santos L. M.. Stochastic Joint Optimization of Wind Generation and Pumped-storage Units in an Electricity Market[J]. IEEE Transactions on Power Systems, 2008, 23(2): 460-468

4. Lin-Her Jeng, Yuan-Yih Hsu, Chang B. S.. A Linear Programming Method for the Scheduling of Pumped-storage Units with Oscillatory Stability Constraints[J]. IEEE Transactions on Power Systems, 1996, 11(4): 1705-1710.

5. Huang Shu, Lin Shunjiang, Liu Mingbo, Multiobjective Security Constrained Dynamic Optimal Dispatch with Wind Farms and Pumped Storage Stations[J]. Proceedings of the CSEE, 2016, 36(1): $112-120$ 